\title{
Desafios e facilidades dos profissionais de educação física nos centros de atenção psicossocial
}

\author{
Challenges and facilities of physical education professionals in psychosocial care centers \\ Desafíos y facilidades de los profesionales de educación física en centros de atención psicosocial
}

\begin{abstract}
RESUMO
Objetivo: Identificar os desafios e facilidades dos profissionais de Educação Física nos Centros de Atenção Psicossocial. Métodos: Trata-se de uma pesquisa de campo, exploratória, com abordagem qualitativa. 0 cenário da pesquisa foi composto pelos Centros de Atenção Psicossocial de um município do Ceará. Os participantes foram os profissionais de Educação Física, destes serviços. A técnica de coleta de dados foi a entrevista estruturada, realizada em novembro de 2013. As entrevistas foram gravadas e posteriormente transcritas para análise. Resultados: Os desafios estão relacionados à desconstrução de paradigmas em relação a categoria e à escassez de materiais e espaços internos para as intervenções. Compromisso dos usuários, formação acadêmica dos profissionais, objeto de estudo da Educação Física e Residências Multiprofissionais em Saúde foram as facilidades identificadas. Conclusão: A atuação destes profissionais é essencial para o cuidado dispensado aos usuários dos serviços de saúde mental e que investimentos devem ser realizados para o desenvolvimento de intervenções exitosas.
\end{abstract}

DESCRITORES: Educação Física; Saúde Mental; Saúde Pública.

\section{ABSTRACT}

Objective: To identify the challenges and facilities of Physical Education professionals in Psychosocial Care Centers. Methods: This is an exploratory field research with a qualitative approach. The research scenario was composed by the Psychosocial Care Centers of a city in Ceará. The participants were the Physical Education professionals of these services. The data collection technique was the structured interview, held in november 2013. The interviews were recorded and later transcribed for analysis. Results: The challenges are related to the deconstruction of paradigms in relation to the category and the scarcity of materials and internal spaces for interventions. Commitment of users, academic training of professionals, object of study of Physical Education and Multiprofessional Residencies in Health were the facilities identified. Conclusion: The performance of these professionals is essential for the care provided to users of mental health services and that investments must be made for the development of successful interventions.

DESCRIPTORS: Physical Education; Mental Health; Public Health.

\section{RESUMEN}

Objetivo: Identificar los desafíos y facilidades de los profesionales de la Educación Física en los Centros de Atención Psicosocial. Métodos: Se trata de una investigación de campo exploratoria con enfoque cualitativo. El escenario de investigación estuvo compuesto por los Centros de Atención Psicosocial de un municipio de Ceará. Los participantes fueron los profesionales de Educación Física de estos servicios. La técnica de recolección de datos fue la entrevista estructurada, realizada en noviembre de 2013. Las entrevistas fueron grabadas y luego transcritas para su análisis. Resultados: Los desafíos están relacionados con la deconstrucción de paradigmas en relación a la categoría y la escasez de materiales y espacios internos para intervenciones. Compromiso de los usuarios, formación académica de los profesionales, objeto de estudio de Educación Física y Residencias Multiprofesionales en Salud fueron las instalaciones identificadas. Conclusión: El desempeño de estos profesionales es fundamental para la atención brindada a los usuarios de los servicios de salud mental y que se deben realizar inversiones para el desarrollo de intervenciones exitosas.

DESCRIPTORES: Educación Física; Salud Mental; Salud Pública.

RECEBIDO EM: 03/05/2021 APROVADO EM: 28/05/2021

\section{Victor Hugo Santos de Castro}

Universidade Estadual do Ceará - UECE - Fortaleza (CE) - Brasil.

ORCID: 0000-0001-7465-9092 


\section{Delane Giffoni Soares}

Universidade Estadual do Ceará - UECE - Fortaleza (CE) - Brasil.

ORCID: 0000-0002-1215-5291

\section{Espedito Afonso Júnior}

Universidade de Fortaleza - UNIFOR - Fortaleza (CE) - Brasil.

ORCID: 0000-0002-3670-251X

\section{Jaqueline Gomes Souza Santos}

Universidade Federal do Ceará - UFC - Fortaleza (CE) - Brasil.

ORCID: 0000-0002-2073-3995

\section{Morgana Nazareth Porfírio de Souza}

Universidade Federal do Ceará - UFC - Fortaleza (CE) - Brasil.

ORCID: 0000-0003-1575-9645

Antônio Elisiário dos Santos Neto

Universidade de Fortaleza - UNIFOR - Fortaleza (CE) - Brasil.

ORCID: 0000-0001-7269-1726

\section{INTRODUÇÃO}

0 s Centros de Atenção Psicossocial (CAPS) são serviços abertos e comunitários de saúde mental, do Sistema Único de Saúde (SUS), que visam atender pessoas com algum tipo de sofrimento mental, incentivando sua integração social e familiar, oferecendo suporte para o desenvolvimento da autonomia no âmbito do trabalho, lazer e outros. Os CAPS constituem a principal estratégia do processo de reforma psiquiátrica ${ }^{(1)}$.

Os profissionais inseridos nos CAPS pertencem a diferentes categorias e integram uma equipe multiprofissional, composta de técnicos de nível superior (enfermeiros, médicos, psicólogos, assistentes sociais, terapeutas ocupacionais, pedagogos, profissionais de Educação Física ou outros necessários para as atividades oferecidas) e de nível médio (técnicos e/ ou auxiliares de enfermagem, técnicos administrativos, educadores e artesãos) ${ }^{(1)}$.

Dentre os profissionais de nível superior supracitados, destacam-se os profissionais de Educação Física, que no âmbito da saúde mental coletiva no estado do Ceará, tem sua inserção fomentada através das Residências Multiprofissionais em Saúde. Estas são orientadas pelos princípios e diretrizes do SUS, a partir das necessidades sócio epidemiológicas da população( ${ }^{(2)}$.

O movimento que a categoria de Educa- ção Física proporciona nos espaços internos dos CAPS e também nos espaços exteriores a estes, é significativo para o cuidado terapêutico ofertado aos usuários ${ }^{(3)}$, colaborando também com a reinserção social, a desinstitucionalização e a humanização( ${ }^{(4)}$.

Algumas possibilidades de intervenção dos profissionais de Educação Física nos CAPS são: atividades aquáticas, atividades rítmicas, alongamento e relaxamento, jogos e brincadeiras, futebol adaptado, futebol livre, vôlei, vôlei adaptado, basquete, tênis, cabo de guerra, circuitos, caminhadas e outros ${ }^{(5)}$.

Considerando o exposto foi elaborado o seguinte questionamento, de cunho investigativo: quais os desafios e facilidades dos profissionais de Educação Física nos CAPS? Portanto, esta pesquisa tem como principal objetivo identificar os desafios e facilidades dos profissionais de Educação Física nos Centros de Atenção Psicossocial.

\section{MÉTODOS}

Trata-se de uma pesquisa de campo, exploratória, com uma abordagem qualitativa. O cenário da pesquisa foi composto pelos Centros de Atenção Psicossocial (geral e álcool e outras drogas) de um município cearense. A amostra foi composta por dois profissionais de Educação Física, os únicos vinculados ao serviço através de uma Residência Multiprofissional em Saúde.
A técnica de coleta de dados foi a entrevista estruturada, realizada em novembro de 2013. A entrevista é um procedimento usado na investigação social, para a coleta de dados ou para identificar, auxiliar ou solucionar um problema social( ${ }^{(6)}$.

$\mathrm{O}$ instrumento de coleta foi um roteiro de entrevista contendo quatro questionamentos, a saber: 1. Qual o maior obstáculo dos profissionais de Educação Física, no âmbito da saúde mental? 2. Quais os seus principais desafios em relação ao desenvolvimento de oficinas de práticas corporais nos CAPS? 3. Qual o principal facilitador para inserção dos profissionais de Educação Física, no âmbito da saúde mental? 4. Quais os maiores facilitadores dos profissionais de Educação Física em relação ao desenvolvimento de oficinas de práticas corporais nos CAPS?

As entrevistas foram gravadas e as falas dos entrevistados foram transcritas para a análise dos dados. Esta foi realizada através da análise de conteúdo, compreendida em três processos, a saber: a pré-análise (leitura e contato com o material de campo); exploração do material (redução textual às expressões significativas); e tratamento dos resultados (relação entre os dados que emergiram na pesquisa e o que se encontra na literatura científica) $)^{(7)}$.

Objetivando garantir o anonimato na pesquisa, os participantes foram identificados e referenciados neste estudo, ale- 
atoriamente, através de números. Cada participante da pesquisa assinou um Termo de Consentimento Livre e Esclarecido (TCLE), com intuito de informar quanto aos benefícios da pesquisa, procedimentos utilizados, objetivos, dentre outros.

$\mathrm{O}$ acesso do pesquisador às organizações para a coleta dos dados foi permitido mediante autorização dos responsáveis por meio da assinatura no Termo de Anuência. O estudo, em seus procedimentos éticos, esteve referenciado pela Resolução $N^{\circ} 466 / 12$ do Conselho Nacional de Saúde (CNS) que se refere às pesquisas com seres humanos ${ }^{(8)}$.

\section{RESULTADOS E DISCUSSÃO}

O primeiro questionamento da entrevista versou sobre o principal obstáculo dos profissionais de Educação Física, no âmbito da saúde mental, especificamente nos CAPS. Os resultados apontam que o principal obstáculo vivenciado e identificado é o paradigma dos outros profissionais de saúde em relação à referida categoria, como pode ser observado nas falas dos entrevistados, a seguir.

"A inserção do profissional de Educação Física nos cenários de saúde mental é recente, logo, alguns entraves são observados. Particularmente, vivencio no CAPS a experiência de ser considerada a profissional que deve dispensar o cuidado em relação ao corpo. Mas através da elaboração de projetos terapêuticos junto à equipe, estou podendo construir o meu espaço junto à comunidade e aos profissionais, mostrando que minha intervenção impacta diretamente na qualidade de vida dos usuários" (Profissional de Educação Física 1).

"Ao chegar ao cenário, foi perceptível que as demandas para os profissionais de Educação Física, eram essencialmente práticas, e quando $n \tilde{a} o$, estavam relacionadas à animação de eventos realizados no próprio CAPS, na visão da gestora $e$ das outras categorias inseridas no serviço. As reunióes de equipe possibilitaram uma discussão sobre as possibilidades de atuação da Educação Física, como debater assuntos específicos nas salas de espera, contribuir no grupo de educação em saúde, a realização de oficinais na perspectiva interprofissional, ter autonomia de realizar o acolhimento do usuário no serviço, mas foi um trabalho árduo, afirmar que eu era um profissional de saúde

\section{Além disso, as \\ intervenções \\ propostas pelos \\ profissionais de \\ Educação Física}

são estímulos

importantes para

que os usuários

do serviço possam

desenvolver novos

hábitos de saúde,

impactando na

qualidade de vida

destes indivíduos no

cotidiano. como todos os outros ali" (Profissional de Educação Física 2).

Corroborando com o exposto, aponta-se que essa aproximação da Educação Física com os serviços de saúde mental, é relativamente recente, como apontado por um participante, destaca-se que para ademais da inserção de outras categorias profissionais, a desconstrução de paradigmas no processo de cuidado terapêutico nos CAPS necessita de rodas de conversa, grupos de convivência, passeios e oficinas terapêuticas ${ }^{(9,10,11)}$, sobretudo, na perspectiva interprofissional, para que os demais profissionais possam identificar as potencialidades da categoria em questão.

Além disso, as intervenções propostas pelos profissionais de Educação Física são estímulos importantes para que os usuários do serviço possam desenvolver novos hábitos de saúde ${ }^{(12)}$, impactando na qualidade de vida destes indivíduos no cotidiano.

A segunda inquirição buscou averiguar quais os principais desafios dos profissionais de Educação Física em relação ao desenvolvimento de oficinas de práticas corporais nos CAPS. Os resultados mostram que os desafios comuns das intervenções propostas estão relacionados à falta de materiais e espaços internos apropriados.

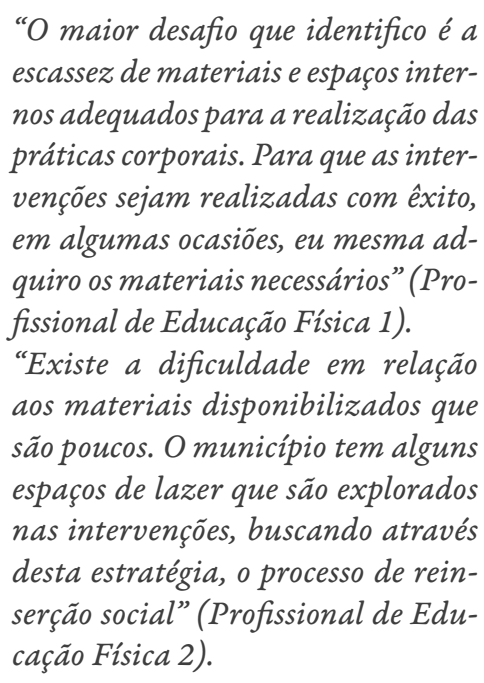

Um obstáculo identificado pelos profissionais de Educação Física nos CAPS é a falta de materiais que impacta diretamente 
na infraestrutura de atendimento dispensada nesses espaços ${ }^{(13)}$. Entretanto, a necessidade de recursos físicos, com os quais os CAPS não contam para a realização de algumas intervenções (práticas corporais), impulsiona experiências em outros espaços da localidade ${ }^{(14)}$. Dessa forma, nota-se que um obstáculo se tornou uma possibilidade de explorar outros territórios de cuidado.

A falta de recursos materiais para as oficinas terapêuticas nos CAPS é recorrente, principalmente pelos processos licitatórios que dificulta a obtenção dos mesmos em tempo favorável ${ }^{(15)}$. Logo, os profissionais devem pensar em alternativas substitutivas, caso a estratégia inicial idealizada, não possa ser realizada ${ }^{(16)}$.

A terceira pergunta sobre o principal facilitador para inserção dos profissionais de Educação Física, no âmbito da saúde mental, especificamente nos CAPS. Os resultados apresentam que a Residência Multiprofissional em Saúde, uma estratégia de educação permanente me saúde, é essencial para que a categoria adentre tais cenários.

"O profissional de educação Física ainda é aquele complementar nos CAPS, mesmo com diversas evidências cientificas de que a atividade física, as práticas corporais ou exercícios físicos têm benefícios significativos para a saúde dos pacientes, então acredito que um facilitador para que esta categoria seja fortalecida nos cenários de saúde mental, é a Residência Multiprofissional em Saúde, pois algumas ofertam vagas para os profissionais de Educação Física na saúde mental, para que a área possam expandir seus horizontes na saúde coletiva" (Profissional de Educação Física 1).

"Posso dizer que nos cenários de saúde da família e comunidade os profissionais de Educação Física já estão consolidados, pois comprovaram através das mais diversas intervençôes que a atividade física é essencial para o enfrentamento de comportamentos de risco, assim como a prevenção de Doenças Crô- nicas não Transmissiveis, mas nos cenários de saúde mental, nossa inserção não é tão comum, então, os programas de residências multiprofissionais que incluem os profissionais de Educação Física nos CAPS, sem dúvidas, são as maiores portas de entrada, para que através das mais diversas contribuiçôes, possamos fortalecer a necessidade da categoria no cuidado dispensado ao paciente de saúde mental" (Profissional de Educação Física 2).

Os programas de Residências Multiprofissionais em Saúde foram regulamentados em 2005 e compreendidos como uma "modalidade de ensino de pós-graduação lato sensu, voltada para a educação em serviço e destinada às categorias profissionais que integram a área de saúde, excetuada a médica"(17). Esta formação objetiva qualificar e motivar os profissionais da saúde para interven ções laborais no SUS e tem duração de dois anos.

Como mencionado trata-se de uma excelente oportunidade para fomentar a inserção dos profissionais de Educação Física nos CAPS. Ressalta-se que a Educação Física não deve ser imposta, mas surgir como uma demanda dos referidos serviços, possibilitando outras formas de organizar o cuidado na saúde mental ${ }^{(3)}$, assim, os programas supracitados tem papel essencial na concretização deste objetivo.

A quarta e última interrogação visou identificar os facilitadores dos profissionais de Educação Física em relação ao desenvolvimento de oficinas de práticas corporais nos CAPS. Os resultados expõem que os facilitadores estão relacionados ao compromisso dos usuários para com as terapias, à formação acadêmica que apontou caminhos para o trabalho colaborativo, ao objeto de estudo da categoria (a cultura corporal do movimento).

"Observo o compromisso que os usuários têm com as oficinas que estamos propondo, estão sempre presentes e são participativos. A Educação Física, através da cultura corporal do movimento, possibilita inúmeras formas de estimular práticas corporais, como esportes, danças, lutas, jogos, ginásticas, brincadeiras e todas estas parecem atrativas para os individuos que frequentam os CAPS" (Profissional de Educação Física 1). "Acredito que a minha formação, abordou a essência do trabalho interprofissional, isso fez com que, eu desenvolvesse oficinas de práticas corporais, incluindo outras categorias profissionais, para que pudéssemos ter diferentes visóes e contribuiçôes das intervençôes, com o objetivo de ofertar um cuidado integral ao usuário. Através disso, consegui desmistificar algumas concepçôes sobre minha categoria" (Profissional de Educação Física 2).

A Educação Física é caracterizada pela cultura corporal do movimento, entendida como um conjunto de conteúdos, que envolve jogos, esportes, ginásticas, danças e lutas ${ }^{(18)}$, possibilitando inúmeras possibilidades de intervenção, considerando inclusive aspectos históricos e regionais, sem dúvidas, atrativas para as terapias.

Quanto ao compromisso dos usuários para com as oficinas realizadas pelos profissionais de Educação Física, pode ser justificado pelo fortalecimento do vínculo entre o profissional e o usuário, favorecendo uma relação de confiança e, sobretudo, mais humanizada, essencial nas intervenções da área da saúde ${ }^{(19)}$.

Pela fala de um dos entrevistados, entende-se que "o cuidado em saúde implica pensar o trabalho em equipe dentro de uma lógica mais abrangente, que demanda investimento coletivo integrado dos diferentes saberes e fazeres em saúde" ${ }^{200}$. Não há profissional que trabalhe individualmente nos CAPS, as intervenções multiprofissionais permitem a interação entre diversas áreas de conhecimento, o que é de extrema eficácia para os tratamentos psicossociais ${ }^{(21)}$.

\section{CONCLUSÃO}

O maior desafio do profissional de 
Educação Física inserido na área de saúde mental é desconstruir os paradigmas dos demais profissionais quanto as suas possibilidades de intervenção. Assim, é essencial que tais profissionais apresentem como a categoria pode impactar positivamente nos comportamentos de saúde dos usuários do serviço, não se limitando às intervenções essencialmente práticas ou de animação.

Outro obstáculo das intervenções é a escassez de materiais e espaços internos para as oficinas de práticas corporais, alertando para a necessidade de investimentos neste quesito. É de suma importância que a gestão do serviço tenha ciência das ne- cessidades e que possa tomar as providências cabíveis, entendo que a continuidade e excelência dos serviços oferecidos pelos CAPS requerem investimentos. Destaca-se que o desafio de espaços internos no CAPS impulsiona a exploração de outros espaços no município, o que é primordial para a reinserção social dos indivíduos.

Quanto às facilidades, destacam-se os programas de Residências Multiprofissionais em Saúde, como uma estratégia que possibilita a inserção dos profissionais de Educação Física na saúde mental, assim como o compromisso dos usuários para com as terapias, à formação acadêmica dos próprios profissionais, o obje- to de estudo da Educação Física para o desenvolvimento de oficinas de práticas corporais nos CAPS.

Ressalta-se que a atuação dos profissionais de Educação Física é essencial para o cuidado dispensado aos usuários dos serviços de saúde mental e que investimentos devem ser realizados para o desenvolvimento de intervenções exitosas. Salienta-se também que esta pesquisa analisou uma realidade específica, apenas um CAPS de um município cearense, assim, recomenda-se estudos posteriores, em outras regiões e realidades, para ampliar a ciência dos desafios e facilidades dos profissionais de Educação Física nos CAPS. -

\section{REFERÊNCIAS}

1. Brasil. Saúde Mental no SUS: os Centros de Atenção Psicossocial. Brasília: Distrito Federal; 2004.

2. Brasil. Portaria Interministerial $n^{\circ} 1.077$, de 12 de novembro de 2009. Brasília: Distrito Federal; 2009.

3. Wachs F. Educação Física e saúde mental: uma prática de cuidado emergente em Centros de Atenção Psicossocial (CAPS) [dissertação]. Porto Alegre: Escola de Educação Física da UFRGS; 2008.

4. Guimarães AC, Pascoal RCA, Carvalho IZ, Adão KS. A inserção social através de práticas de educação física como medidas interventivas para pacientes psicóticos e neuróticos graves do CAPS de São João del-Rei. Revista Pesquisas e Práticas Psicossociais. 2012; 7(2): 254-259.

5. Castro VHS; Abreu SMB. Educação Física e Saúde Mental: Oficinas de Práticas Corporais como Estratégia de Cuidado nos Centros de Atenção Psicossocial. Revista de Educação Física, Saúde e Esporte. 2019; 2(1): 40-55.

6. Lakatos EM, Marconi MA. Fundamentos de Metodologia Científica. 4.ed. São Paulo: Atlas; 2001.

7. Minayo MCS. O desafio do conhecimento: pesquisa qualitativa em saúde. São Paulo: Hucitec; 2007.

8. Brasil. Resolução N 466. 2012dez [cited 2021 mar 15]. Available from: https://bvsms.saude.gov.br/bvs/saudelegis/ cns/2013/res0466_12_12_2012.html.

9. Ramminger T, Brito JC. Cada CAPS é um CAPS: uma coanálise dos recursos, meios e normas presentes nas atividades dos trabalhadores de saúde mental. Revista Psicologia \& Sociedade. 2011; 23: 150-160.

10. Cadore C, Beck CLC. O processo terapêutico em um CAPSad: a visão dos trabalhadores. In: Anais da jornada de Pesquisa em Psicologia. UNISC, 2011.

11. Campos GWS. Saúde Paidéia. In: Saúde em debate. São Paulo: Hucitec; 2003.
12. Oliveira CS et al. O profissional de Educação Física e sua atuação na saúde pública. EFDeportes.com. 2011: 15(153).

13. Kantorski LP, Coimbra VCC, Silva ENF, Guedes AC, Cortes JM, Santos F. Avaliação qualitativa de ambiência num Centro de Atenção Psicossocial. Revista Ciência e Saúde Coletiva. 2011; 16(4): 2059-2066.

14. Wachs F, Fraga AB. Educação Física em Centros de Atenção Psicossocial. Revista Brasileira de Ciências do Esporte. 2009; 31(1): 93-107.

15. Machado GJ. A atuação do professor de educação física nos centros de atenção psicossocial álcool e drogas da grande Vitória-ES [dissertação]. Vitória: Centro de Educação Física e Desporto da UFES; 2015.

16. Ferreira LAS. O trabalho da Educação Física na composição de equipe de saúde mental especializada em álcool e outras drogas [dissertação]. Porto Alegre: Escola de Educação Física da UFRGS; 2013.

17. Brasil. Lei 11.129. 2005jun [cited 2021 mar 15]. Available from: http://www.planalto.gov.br/ccivil_03/_ato20042006/2005/lei//11129.htm.

18. Darido CS, Rangel ACI. Educação Física na Escola. Rio de Janeiro: Guanabara Koogan; 2005.

19. Furtado RP, Oliveira MFM, Sousa MF, Vieira PS, Neves RLR, Rios GB. O trabalho do professor de educação física no CAPS: aproximações iniciais. Revista Movimento. 2015; 21(1): 41-52.

20. Ferreira LAS, Damico JGS, Fraga AB. Entre a composição e a tarefa: estudo de caso sobre a inserção da Educação Física em um serviço de Saúde Mental. Revista Brasileira de Ciências do Esporte. 2017; 39(2): 176-182.

21. Muylaert CJ. Formação, vida profissional e subjetividade: narrativas de trabalhadores de Centro de Atenção Psicossocial Infanto-juvenil [dissertação]. São Paulo: Faculdade de Saúde Pública da Universidade de São Paulo; 2013. 